\title{
Spontaneous mentalizing in neurotypicals scoring high versus low on symptomatology of autism spectrum disorder
}

\author{
Annabel D. Nijhof, Marcel Brass, Jan R. Wiersema
}

\begin{abstract}
Spontaneous mentalizing ability has been linked to symptoms severity in individuals with autism spectrum disorder (ASD). Here we investigated whether in neurotypicals, higher levels of ASD symptomatology could also be linked to lower levels of spontaneous mentalizing, by comparing neurotypicals scoring high with those scoring low on the short Autism Spectrum Quotient. Participants watched movies during which they, and another agent, formed beliefs about the location of an object. These beliefs could influence reaction times (RT) to that object in the outcome phase. We expected participants with more ASD symptoms to show less spontaneous mentalizing, as reflected by a smaller effect of the other agent's beliefs on RT patterns (the ToM index). In contrast, the effect of own beliefs on RTs, reflecting an egocentric bias, was expected to be larger in the high-scoring group. Results showed that groups differed in the effect of the agent's beliefs; the ToM index was highly significant in the low-scoring group, while being absent in the highscoring group. No difference in egocentric bias was observed. These findings suggest that the relationship between levels of ASD symptomatology and spontaneous mentalizing is not only present in individuals with ASD, but also in the neurotypical population.
\end{abstract}

\section{Introduction}

Theory-of-Mind (ToM), also referred to as mentalizing, is defined as the ability to attribute mental states (such as desires, beliefs or intentions) to oneself or others (Premack and Woodruff, 1978; Wimmer and Perner, 1983). This ability is thought to underlie successful communication and social interaction. Because individuals with autism spectrum disorder (ASD) show deficits in exactly these capacities as a crucial part of their symptomatology (American Psychiatric Association, 2013), researchers have argued that ASD is characterized by a specific ToM deficit (Baron-Cohen et al., 1985; Rajendran and Mitchell, 2007). ToM ability has been investigated mostly with 'false-belief tasks': tasks in which an agent holds a false belief about the location of an object, because it was moved outside of the agent's awareness. Participants are asked where the agent will search for this 
object, and when they correctly take into account the agent's false belief, this is seen as successful ToM.

Based on studies with these false-belief tasks, for a long time ToM was thought to develop around the age of four years (Wellman et al., 2001). However, more recently, when using different measures of mentalizing, such as eye-tracking, that do not require language or other higher cognitive skills, evidence for mentalizing was found in children much younger than 4 years (Onishi and Baillargeon, 2005; Senju et al., 2011; Southgate et al., 2007; Surian et al., 2007), for one study even as young as only seven months old (Kovács et al., 2010). For this reason, Apperly and Butterfill proposed the 'two-systems account of mentalizing' (Apperly and Butterfill, 2009). They hypothesized that there are two mentalizing systems: one system entails an implicit or spontaneous form of mentalizing that develops early, and which is fast and inflexible; the other is a more explicit form of mentalizing developing at a later age, which is more cognitively demanding and slow, but also more flexible. It has been debated whether there are really two separate systems, or whether there is one core mentalizing system, which can either operate spontaneously or, under more controlled conditions, in combination with additional domain-general resources such as executive functioning and working memory (Carruthers, 2015). This latter view is supported by the recent finding that the brain regions underlying both forms of mentalizing overlap to a great extent (Bardi et al., 2016; Van Overwalle and Vandekerckhove, 2013). Bardi et al. (2016) directly compared a spontaneous and an explicit version of a ToM task, which is the same task that we will apply in the current study. During this task, both spontaneous and explicit belief processing activated the medial prefrontal cortex and right temporo-parietal junction, two regions that have consistently shown to activate during explicit mentalizing (Decety and Lamm, 2007; Schurz et al., 2014; Van Overwalle, 2009).

In any case, the existence of a spontaneous form of mentalizing might help to resolve the controversies surrounding the 'ToM theory of ASD'. Soon after Baron-Cohen introduced the theory of a specific ToM deficit in ASD (Baron-Cohen et al., 1985), it was already criticized because a relatively large amount of children and adults with ASD, especially high-functioning individuals, passed ToM tests (Bowler, 1992; Frith and Happé, 1994; Ozonoff et al., 1991). Since then, studies have shown that individuals with ASD can even succeed on more advanced ToM tasks (Scheeren et al., 2013; Spek et al., 2010). Still, they do show profound difficulties with everyday social communication and interaction. The argument would be that these individuals have a deficit in spontaneous mentalizing, but use compensatory strategies on explicit mentalizing tasks: in order to solve these tasks, they apply learnt rules and, if intact, their executive functioning skills, thus masking their reduced ability to mentalize spontaneously (Frith, 2004; Ozonoff et al., 1991; Pellicano, 2010).

Several studies to date have indeed found support for impaired spontaneous mentalizing in people with ASD (Callenmark et al., 2013; Deschrijver et al., 2015; Schneider et al., 2013; Schuwerk et 
al., 2015; Senju, 2013; Senju et al., 2009). However, all of these studies have taken a categorical approach to ASD, comparing individuals with a diagnosis to neurotypical controls. More and more, researchers are taking a dimensional approach to developmental psychopathology (Hudziak et al., 2007), arguing that individuals with a diagnosis are at the end of a continuum of traits existing in the general population, and that creating a categorical dichotomy will inevitably lead to the loss of potentially interesting information. Additionally, studying the non-clinical population has the advantage of reducing the influence of comorbidities present in the clinical population, such as attention-deficit hyperactivity disorder, anxiety and depression in the case of ASD (Joshi et al., 2013; Mannion and Leader, 2013). These arguments have been recognized in the field of ASD specifically, where researchers acknowledge the importance of taking into account the presence of ASD-related behavior and personality traits in relatives of individuals with ASD (the broader autism phenotype or BAP) (Losh et al., 2011; Parr and Le Couteur, 2013), as well as in the neurotypical population more generally (Constantino, 2011; Constantino and Todd, 2003; Robertson and Simmons, 2013; Robinson et al., 2011).

In this light, it would be interesting to investigate whether the link between spontaneous mentalizing ability and ASD symptomatology is also present in the neurotypical population. Therefore, with the current study we wanted to see if we would find differences in spontaneous mentalizing between people scoring high versus low on ASD symptomatology in the neurotypical population. In order to measure spontaneous mentalizing, we used the 'Buzz Lightyear task', a simple ball detection task based on the study by Kovács and colleagues (2010). Within this task, both the participants themselves and another agent (Buzz) form a belief about the location of a ball, but they are never asked about these beliefs explicitly. By recording reaction times to ball presence, which can be expected or unexpected for the participant and/or the other agent, one can measure the extent to which participants spontaneously tracked their own and other's beliefs. A crucial measure in this task is the so-called 'ToM index': the difference between the condition in which neither participant nor Buzz expect the ball and that in which only the agent expects it: if RTs are faster only on the basis of what the other agent was expecting, this can be taken as evidence for spontaneous mentalizing.

Recently, the Buzz Lightyear task has been validated both in neurotypical and ASD samples (Deschrijver et al., 2015; Nijhof et al., 2016), and in the fMRI study mentioned previously (Bardi et al., 2016). Deschrijver et al. (2015), who applied the task in ASD, found that adults with high-functioning autism (HFA) had a significantly larger egocentric bias in spontaneous belief processing, reflecting an increased adherence to their own beliefs/expectations, as has also been reported in literature on explicit ToM (Begeer et al., 2012; Fisher et al., 2005; Williams and Happé, 2009). In addition, the effect of the other agent's belief, as reflected in the ToM index, was found to be correlated negatively with ASD symptomatology within the HFA group, indicating less spontaneous mentalizing 
with higher levels of symptomatology. This latter finding supports the hypothesis that the relationship between spontaneous mentalizing and ASD traits may be seen as a continuum rather than as a categorical distinction between people with and without an ASD diagnosis.

In a recent study, we compared performance on an explicit and implicit spontaneous version of the Buzz Lightyear task in a neurotypical sample (Nijhof et al., 2016). RT patterns were similar for both versions, and the presence of the ToM index showed that participants indeed calculated the belief of another agent spontaneously (i.e., without being instructed to do so). In contrast to the study of Deschrijver et al. (2015), measures of ASD symptomatology in this study did not correlate with the size of the ToM index. This may, however, have been due to restricted variation in ASD symptoms within the neurotypicals included in the study. To address this, in the current study, we compared neurotypical participants with high and low extremes of ASD traits. This allows us to investigate whether the inverse relationship between spontaneous mentalizing and ASD traits is specific to the ASD population, or can be observed more broadly. The hypothesis would be that individuals scoring high on ASD symptoms show less spontaneous mentalizing than those scoring low, which would be indicative of a specific relationship between ASD traits and the ability to mentalize spontaneously. In other words, we expected the ToM index to be present in the lowscoring group, and it to be decreased or absent in the high-scoring group. In addition, in line with the findings of Deschrijver et al. (2015), we expected to find a larger effect of own belief in our group scoring high on ASD measures, reflecting an egocentric bias. 


\section{Methods}

\subsection{Participants}

Participants were contacted through Experimetrix, the online system of Ghent University used to recruit students for experimental research. As part of this system, participants already filled out a large battery of short screening questionnaires, including the AQ-10 (Allison et al., 2012), a screener for autism symptomatology which consists of the 10 items with highest sensitivity and specificity of the 50-item Autism Spectrum Quotient (AQ; Baron-Cohen et al., 2001). Based on the online database of all AQ-10 scores ( $N=427)$, a cut-off for the highest and lowest $20 \%$ of scores was set. Students with a score lower than 2 fell in the bottom $20 \%$ range and were recruited for the lowscoring group, while students with a score of 5 or higher were recruited for the high-scoring group. This resulted in a group of 31 participants in the high AQ-10 range, and 29 participants in the group scoring low on the AQ-10. None of the participants in either group reported any history of neurological or psychiatric disorders (including an ASD diagnosis). All participants gave informed consent prior to the study, which was approved by the local ethics committee of the Faculty of Psychology and Educational Sciences of Ghent University, and received a financial reward for their participation.

Data of one participant in the low-scoring group were not saved correctly, so as a result, our final sample consisted of 31 participants in the 'high-AQ' group (7 male, 5 left-handed), and 28 participants in the 'low-AQ' group ( 2 male, 2 left-handed). Mean group characteristics (including between-group comparisons) are displayed in Table 1.

To check for the reliability of the AQ-10 score, during the experimental session participants also filled out the full AQ and the Social Responsiveness Scale for adults (SRS-A; Constantino and Gruber, 2002), which is another self-report screening questionnaire of ASD symptomatology, primarily addressing social responsiveness. The mean full AQ score across the two groups was 15.0 ( $S D=8.6$ ), in line with previous findings in neurotypical populations (Ruzich et al., 2015). Mean SRS-A score was 41.1 (SD = 21.7), which is also comparable to previous findings (Ingersoll et al., 2011). In confirmation of the validity of the created groups, both full AQ and SRS-A score differed significantly between groups(see Table 1). In addition, a Spearman's correlation revealed that both full AQ score and SRS-A score correlated with participants' AQ-10 score across groups (full AQ: $\rho=0.60, p<.001$; SRS-A: $\rho=0.52, p<.001)$.

\section{Table 1}


Group characteristics of the group scoring high and the group scoring low on the AQ-10, and their statistical comparisons between the two groups.

\begin{tabular}{llll}
\hline & $\begin{array}{l}\text { High-AQ group } \\
\text { Mean (Standard } \\
\text { deviation) }\end{array}$ & $\begin{array}{l}\text { Low-AQ group } \\
\text { Mean (Standard } \\
\text { deviation) }\end{array}$ & $\begin{array}{l}\text { Between-group } \\
\text { comparison }\end{array}$ \\
\hline Age (years) & $22.5(4.5)$ & $22.0(2.6)$ & $\mathrm{t}(57)=0.50, \mathrm{p}=.62$ \\
Sex & 7 male, 24 female & 2 male, 26 female & $\chi^{2}(1)=2.89, \mathrm{p}=.09$ \\
Full AQ score & $20.0(7.8)$ & $9.7(5.9)$ & $\mathrm{t}(57)=5.55, \mathrm{p}<.001$ \\
SRS-A score & $51.5(18.5)$ & $30.1(19.4)$ & $\mathrm{t}(57)=4.24, \mathrm{p}<.001$
\end{tabular}

\subsection{Task}

The implicit version of the recently developed Buzz Lightyear task (Bardi et al., 2016; Deschrijver et al., 2015; Nijhof et al., 2016), an adaptation of a similar task by Kovács et al. (2010), was used. For a detailed description of the task, see also Nijhof et al. (2016). During the Buzz Lightyear task, participants watched short videos (13,850 milliseconds; 720 by 420 pixels), presented on a black background using Presentation software (version 18.3). See supplementary material for an example video (note that the video may be of lower quality due to formatting).

During these videos both the participants themselves, and another agent (Buzz Lightyear) formed a belief about the location of a ball, but they were never explicitly asked about these beliefs. The exact instructions were as follows (literal translation from Dutch): "You are asked to carry out a visual detection task. Instructions: Press the left response button (' $V$ ') when Buzz leaves the scene. Press the right response button (' $B$ ') as soon as possible only if the ball is present when the green occluder falls."

In each video, participants saw Buzz Lightyear placing a ball on a table, after which the ball started rolling around. First, the ball rolled behind a green occluder, and subsequently there were four possible continuations (Belief Formation phase, Figure 1):

1. Resulting in the other agent (Buzz) holding a true belief (i.e., true in the eyes of the participant) about the ball being present behind the occluder $(\mathrm{P}+\mathrm{A}+$ condition: $\mathrm{P}=$ participant, $\mathrm{A}=$ agent, $+=$ belief of presence, - = belief of absence).

2. Resulting in the agent holding a true belief about the ball being absent, meaning the ball had rolled out of the scene instead of behind the occluder (P-A-condition).

3. Resulting in the agent holding a false belief about the ball being present (P-A+ condition).

4. Resulting in the agent holding a false belief about the ball being absent ( $P+A-$ condition). In each video, Buzz left the scene at some point (5000 ms after movie onset for condition P-A+, 7624 ms after movie onset for condition $\mathrm{P}+\mathrm{A}-, 9874 \mathrm{~ms}$ after movie onset for conditions $\mathrm{P}+\mathrm{A}+$ and $\mathrm{P}-\mathrm{A}-\mathrm{-}$. At 
this point participants had to press the left button (' $V$ ' on the keyboard), in order to assure they were paying attention to the ongoing video. After Buzz had left, the ball could either stay in the same location ( $\mathrm{P}+\mathrm{A}+$ and $\mathrm{P}-\mathrm{A}$ - condition), or change location, which lead to the agent holding a different belief than the participant ( $\mathrm{P}+\mathrm{A}-$ and $\mathrm{P}-\mathrm{A}+$ condition).

At $12,694 \mathrm{~ms}$ in the video, the agent returns and at $13,250 \mathrm{~ms}$, the occluder falls down. This is the Outcome phase (see Figure 1): on half of the trials, the ball is present $(\mathrm{B}+$ ) behind the fallen occluder. On these trials, participants were instructed to press the right button ('B' on the keyboard) as quickly as possible. On the other half of the trials, where the ball is not present (B-), they should not press the button. Absence or presence of the ball is completely random and independent of the Belief Formation phase. In previous research with this task (Bardi et al., 2016; Nijhof et al., 2016), debriefing questionnaires confirmed that participants were unaware of the manipulation of Buzz' belief, and mentalizing can therefore indeed be considered spontaneous.

In total, the task thus consists of 8 conditions: 2 (participant's belief: present or absent; $\mathrm{P}+$ or P-) $x 2$ (agent's belief: present or absent; A+ or A-) $\times 2$ (outcome: ball present or absent; B+ or B-), with reaction time data available for half of these conditions (i.e., the $\mathrm{B}+$ conditions).

Participants started with four practice trials, for which feedback was presented. After this, the actual task started and trials were presented without feedback, with an ITI of 1000 ms between movies. Each of the eight different movies was presented six times, resulting in 48 trials in total. These were presented randomly in two blocks of 24 trials with a short break in between. 

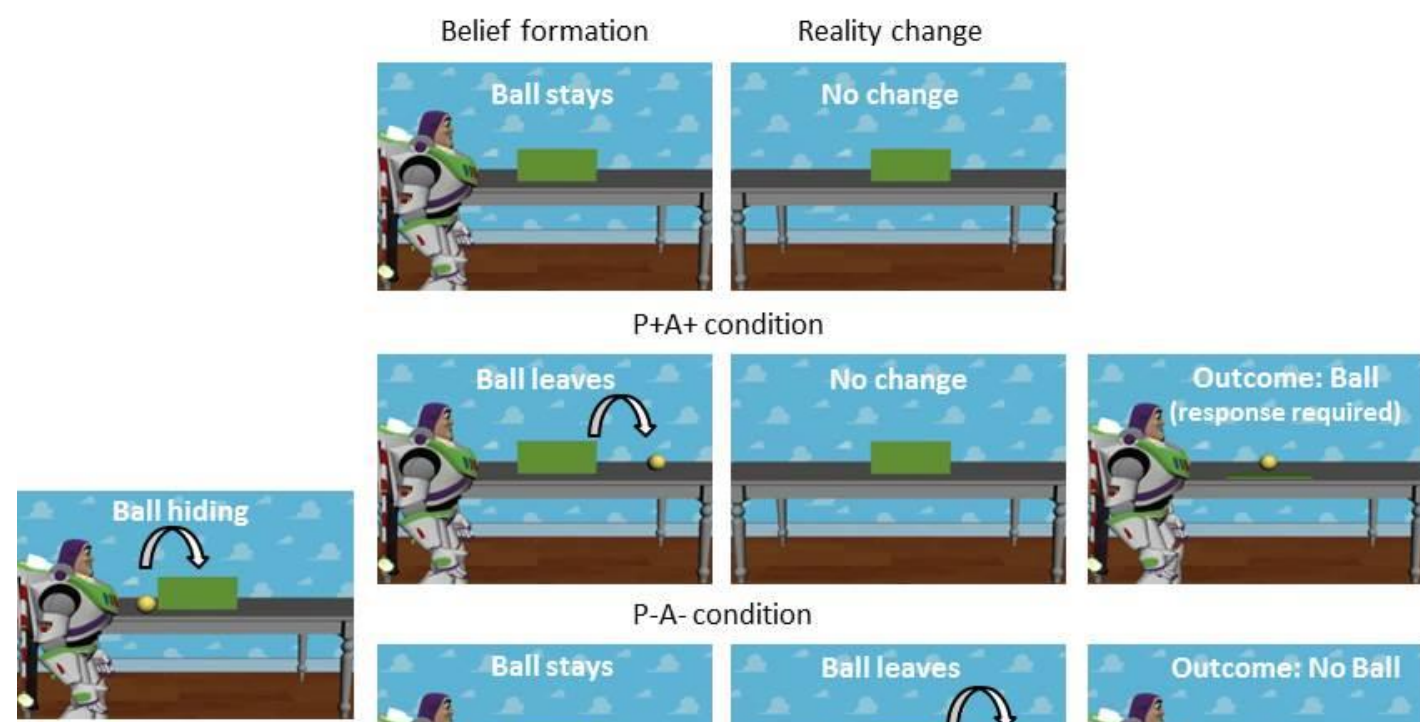

P-A-condition
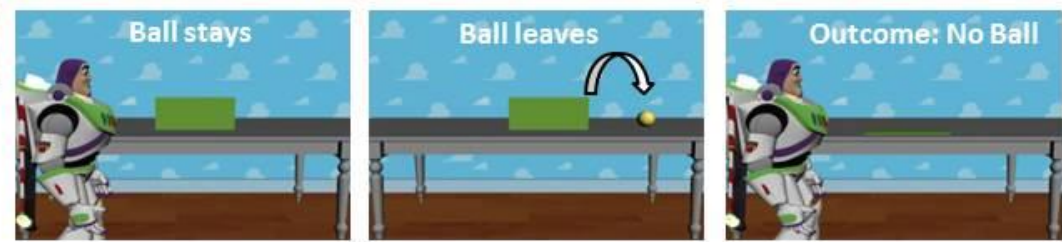

P-A+ condition

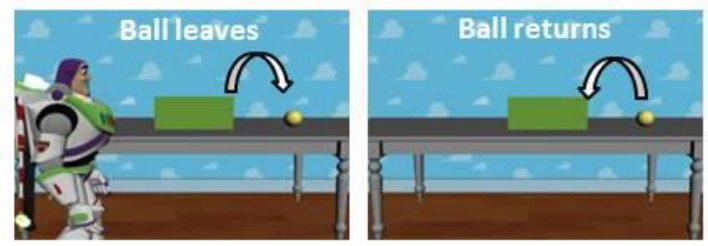

$\mathrm{P}+\mathrm{A}$ - condition

Figure 1. Schematic illustration of the eight different conditions. $P=$ participant, $A=$ agent, $+=$ expecting the ball, - = not expecting the ball. Eight conditions result from four possibilities in the Belief Formation phase $(B+C$, middle) and two possibilities (ball / no ball) in the Outcome phase ( $D$, right). The Belief Formation phase and Outcome phase are independent from each other. In the first part of the movie (A), the ball rolls behind the occluder. In the second part (B), Buzz is present and the ball sometimes changes location. Between B and C, Buzz disappears and a left button response is required to indicate Buzz leaving the scene. In the third part (C), Buzz is absent and the ball sometimes changes location. In the last part (D; the Outcome phase), the occluder falls and the ball is present or not. Only when the ball is present, a right button response is required. (Adapted from Nijhof et al., 2016). 


\subsection{Procedure}

Participants filled out the $A Q-10$ questionnaire online, and came to the university for the experimental session. The Buzz Lightyear task was performed first, and took about 15 minutes. After this, two other short experimental tasks were carried out, which will be reported elsewhere. Finally, participants filled out the full AQ and SRS-A questionnaire. In total, the experimental session lasted approximately one hour per participant.

\subsection{Data analysis}

All statistical analyses were conducted with IBM SPSS Statistics 20 (SPSS Inc., Chicago, IL, USA). For the reaction time data, outliers of more than three standard deviations above or below the mean were removed per participant, as well as responses that were slower than $1000 \mathrm{~ms}$ or faster than 100 ms. A repeated measures ANOVA was performed on the remaining correct reaction times (trials with a ball), with factors of Own Belief (present/absent), Other's Belief (present/absent) and Group (high $A Q$, low $A Q$ ). In this way, we could investigate effects of spontaneous mentalizing as well as a possible egocentric bias, within and between groups. As we were particularly interested in the ToM index, planned comparisons were carried out between the crucial P-A+ and P-A-conditions.

For all analyses, estimates of effect size were reported: partial eta-squared was reported for the ANOVA (0.01 = small, $0.06=$ medium, $0.14=$ large effect $)$, and Cohen's $d(0.2=$ small, $0.5=$ medium, 0.8 = large effect) for t-tests (Cohen, 1988).

\section{Results}

\subsection{Accuracy}

Accuracy was very high for both groups: in the low-AQ group, participants only made 0.9 misses on average on the 24 trials with the ball, and 1.5 false alarms on the 24 trials without the ball. In the high-AQ group, these were 2.0 misses and 1.5 false alarms on average. Because of the small number of trials, this gives too little data to perform reliable group analyses. For this reason, no further analyses were performed on accuracy data.

\subsection{Reaction time}

Average RTs per condition are displayed per group in Figure 2. The ANOVA with factors Own Belief, Other's Belief and Group revealed a main effect of Group $(t(57)=2.21, p=.03, d=0.59$ ): across conditions, average RT was higher in the group scoring high on $A Q-10(M=352.4, S D=9.2)$ than in the low-scoring group $(M=322.8, S D=9.7)$. In addition, there was a main effect of Own 
Belief $\left(F(1,57)=27.40, p<.001, \eta^{2}=0.33\right)$. This effect (the 'egocentric bias') can be explained as RTs being faster for those conditions in which the participants were expecting the ball $(P+A+$ and $P+A-$; mean $R T=327.2)$ than in conditions in which they were not $(P-A+$ and $P-A-;$ mean $R T=348.0)$. This effect was not significantly different between groups $\left(F(1,57)=0.01, p=.93, \eta^{2}=0.00\right)$.

Also, there were two significant interaction effects. First, there was an interaction between Own and Other's Belief $\left(F(1,57)=14.65, p<.001, \eta^{2}=0.21\right)$. A planned comparison showed that RTs to P-A+ were significantly shorter than to P-A- (the 'ToM index', $p=.001$ ). Second, important for our hypothesis, Other's Belief interacted with Group $\left(F(1,57)=6.23, p=.02, \eta^{2}=0.10\right)$. Post hoc testing demonstrated that the effect of Other's Belief was present in the low AQ-group $(F(1,27)=5.49, p=$ $\left..03, \eta^{2}=0.17\right)$, but absent in the high-AQ group $\left(F(1,30)=1.52, p=.23, \eta^{2}=0.05\right)$.

Finally, in order to test our main hypothesis, we evaluated the size of the ToM index (the difference between $\mathrm{P}-\mathrm{A}-$ and $\mathrm{P}-\mathrm{A}+$ ) in both groups. The ToM index turned out to be highly significant in the low-AQ group $(p=.001)$, while it was not present in the high-AQ group $(p=.20)$. In the low-AQ group, it was $26.91 \mathrm{~ms}$ on average ( $S D=38.87)$, and 9.64 in the high-AQ group (SD $=40.77)$. A direct comparison of the ToM index between groups showed a marginally significant difference (t $(57)=$ $1.66, p=.10, d=0.44)$. Importantly, sensitivity analyses including sex as a covariate did not alter the significance of our findings.

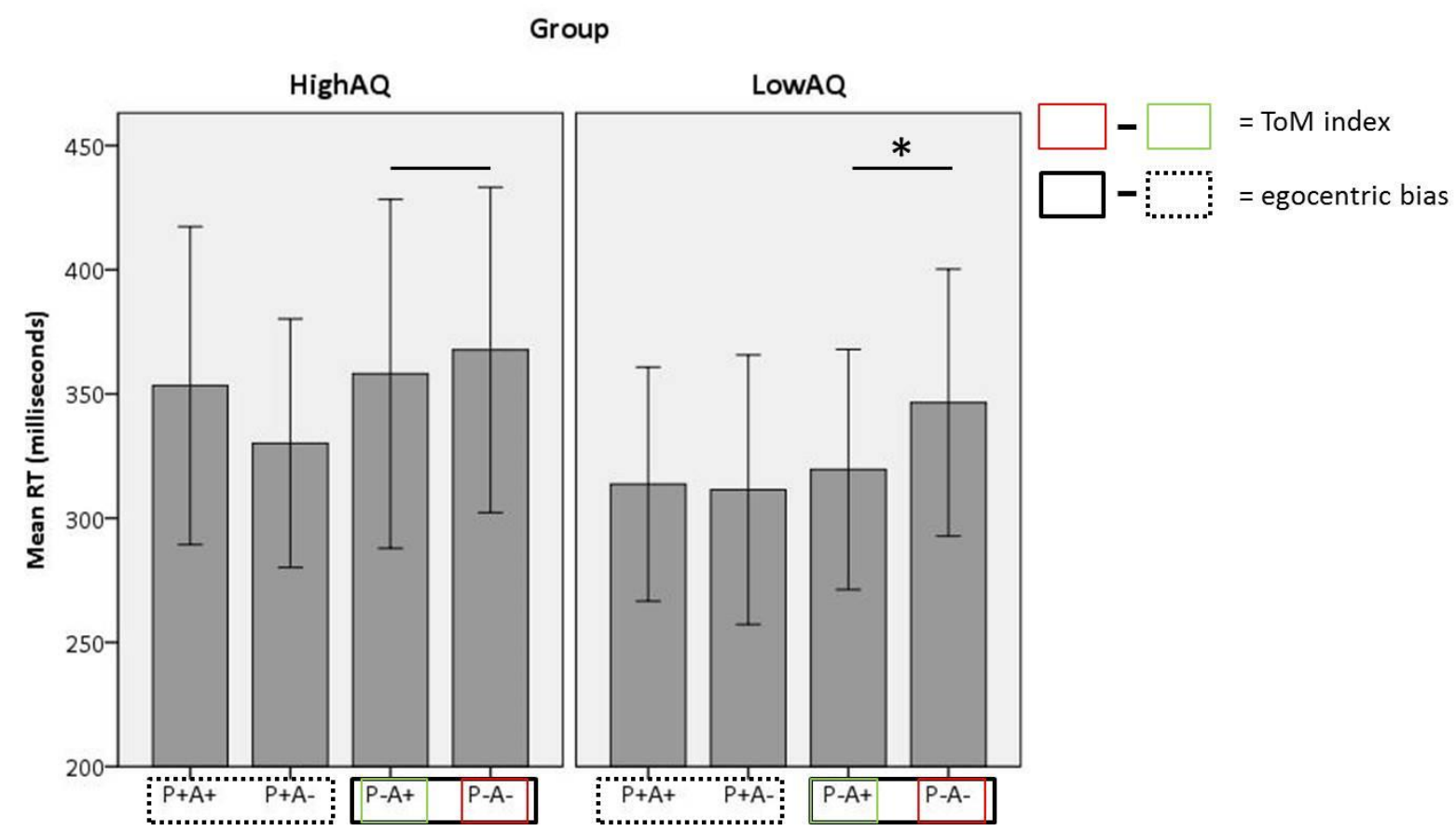

Figure 2. Average reaction times in milliseconds (+/- 1 standard deviation) per condition, per group. $P$ $=$ participant, $\mathrm{A}=$ agent,$+=$ expecting the ball, $-=$ not expecting the ball. The relevant comparisons are indicated in the figure legend. The asterisk shows that the planned comparison for the ToM index is significant in the low-AQ group (and not the high-AQ group). 


\section{Discussion}

Several recent studies have suggested that individuals with ASD show a specific deficit in spontaneous mentalizing (Callenmark et al., 2013; Deschrijver et al., 2015; Schneider et al., 2013; Schuwerk et al., 2015; Senju, 2013; Senju et al., 2009). To date, it was unclear whether this reflects a categorical distinction between people with an ASD diagnosis and neurotypicals. However, the results of the study by Deschrijver et al. (2015) suggested that ASD symptom severity contributes to the effect, as within their ASD group, individual differences in ASD severity were inversely correlated with the level of spontaneous mentalizing. This finding indicates that at least within the ASD population, individuals can vary in the extent to which they are impaired in spontaneous mentalizing, and that this is linked to the amount of ASD behavior and traits they exhibit. With the current study, we wanted to test whether the same would hold for the neurotypical population.

Using the validated 'Buzz Lightyear task' we showed, for the first time, that differences in spontaneous belief processing were indeed related to self-report measures of ASD symptomatology in a neurotypical sample as well. Selecting neurotypical participants based on extreme scores on the AQ-10, we created groups of adults scoring high versus low on self-reported ASD traits. Replicating previous findings with the same task (Bardi et al., 2016; Deschrijver et al., 2015; Nijhof et al., 2016), we found that both the effect of participants' own belief and the interaction of this effect with the other agent's belief significantly influenced reaction times, with RTs being longest for the condition in which neither the participant nor Buzz was expecting the ball. The difference between this condition $(\mathrm{P}-\mathrm{A}-)$ and the condition in which only Buzz was expecting the ball $(\mathrm{P}-\mathrm{A}+)$ has been referred to as the ToM index in previous studies. Crucially, the ToM index was indeed present in our results, meaning that participants were significantly faster in responding to the ball in the condition where only Buzz expected the ball than where neither the participant nor Buzz expected the ball.

Focusing on group differences, first of all, we found that participants in the high-AQ group were overall slower in detecting the ball, as indicated by a main group effect on RT. In clinical ASD samples, slower detection RTs have been observed in previous studies (Ciesielski et al., 1990; Schmitz et al., 2007), that have been associated with differences in selective attention and attention switching (Allen and Courchesne, 2001). Our findings indicate that slower detection is also evident in those neurotypicals who score high on ASD traits.

More importantly though, in line with our hypotheses, groups differed significantly on the effect of the other agent's belief on reaction time patterns, with the group scoring high on ASD symptomatology showing a decreased effect of the other agent's belief on RTs. This is indicative of a 
link between spontaneous mentalizing and ASD symptomatology not only in the ASD population (Deschrijver et al., 2015), but also in the neurotypical population.

Most previous studies using this paradigm focused at the ToM index to measure spontaneous mentalizing (Bardi et al., 2016; Deschrijver et al., 2015; Kovács et al., 2010; Nijhof et al., 2016), which is argued to be the purest reflection of the effect of the other's belief on RT. The argument is that if RTs are faster only on the basis of what the other agent was expecting, this can be taken as evidence for spontaneous mentalizing. Further testing in our study indeed showed that the ToM index was highly significant for the low-AQ group, while this effect was absent in the high-AQ group. It has to be noted though that there was a large inter-individual variability on this measure in both samples. Further studies are needed to address the issue of inter-individual variability and to replicate our findings in a larger sample, as this will increase power and may thus provide a more conclusive answer.

One could argue that using a realistic human agent, rather than the cartoon figure Buzz, would provide a more ecologically valid representation of our everyday social interactions with others. However, previous studies with adults have validated the Buzz Lightyear task as a reliable measure of spontaneous mentalizing, both at the behavioral and at the neural level (Bardi et al., 2016; Deschrijver et al., 2015; Nijhof et al., 2016), and our current findings add to this. Nevertheless, it would be an interesting question for future research whether using a more realistic other agent would give stronger effects.

The results also revealed an effect of own beliefs on detection speed. However, no group difference was found for the effect of own belief, contrasting the finding by Deschrijver et al. (2015) of a larger egocentric bias in their ASD group. Possibly this could be taken to indicate that rather than a dimensional trait, the increased egocentric bias, reflecting a stronger-than-typical adherence to own beliefs/expectations, is a categorical distinction between individuals with and without ASD.

In short, with our study we found evidence for a negative relationship between self-reported ASD symptoms and spontaneous mentalizing ability (ToM index) in a neurotypical sample, adding to similar findings in a sample of adults with high-functioning ASD (Deschrijver et al., 2015). These results once again emphasize the importance of studying cognitive functions that show a specific link to ASD-related behavior and traits in the neurotypical population as well. Firstly, this allows researchers to investigate these functions without the disadvantages of running clinical studies, and secondly, findings in the neurotypical population can inform researchers about the specificity of certain deficits in the ASD population. In fact, these arguments do not only hold for cognitive functions, but also for biological and neurological functions. Since spontaneous mentalizing has recently been linked to activity in the TPJ and mPFC (Bardi et al., 2016; Kovács et al., 2014), two regions typically involved in explicit mentalizing (Decety and Lamm, 2007; Schurz et al., 2014; Van 
Overwalle, 2009), it would be interesting to see whether activity in these regions is also related to measures of ASD symptomatology both within individuals with and without an ASD diagnosis.

Funding: This work was supported by the Special Research Fund (BOF) of Ghent University, project number $\mathrm{B} / 13472 / 01$. 


\section{References}

Allen, G., Courchesne, E., 2001. Attention function and dysfunction in autism. Front. Biosci. a J. virtual Libr. 6, D105-19.

Allison, C., Auyeung, B., Baron-Cohen, S., 2012. Toward brief "red flags" for autism screening: The short Autism Spectrum Quotient and the short Quantitative Checklist in 1,000 cases and 3,000 controls. J. Am. Acad. Child Adolesc. Psychiatry 51, 202-212. doi:10.1016/j.jaac.2011.11.003

American Psychiatric Association, 2013. Diagnostic and Statistical Manual of Mental Disorders (5th ed.). Author, Washington, DC.

Apperly, I.A., Butterfill, S.A., 2009. Do humans have two systems to track beliefs and belief-like states? Psychol. Rev. 116, 953-970. doi:10.1037/a0016923

Bardi, L., Desmet, C., Nijhof, A.D., Wiersema, J.R., Brass, M., 2016. Brain activation for spontaneous and explicit false belief tasks overlaps: new fMRI evidence on belief processing and violation of expectation. Soc. Cogn. Affect. Neurosci. nsw143. doi:10.1093/scan/nsw143

Baron-Cohen, S., Leslie, A.M., Frith, U., 1985. Does the autistic child have a "theory of mind" ? Cognition 21, 37-46. doi:10.1016/0010-0277(85)90022-8

Baron-Cohen, S., Wheelwright, S., Skinner, R., Martin, J., Clubley, E., 2001. The Autism Spectrum Quotient : Evidence from Asperger syndrome/high functioning autism, males and females, scientists and mathematicians. J. Autism Dev. Disord. 31, 5-17.

Begeer, S., Bernstein, D.M., van Wijhe, J., Scheeren, a. M., Koot, H.M., 2012. A continuous false belief task reveals egocentric biases in children and adolescents with Autism Spectrum Disorders. Autism 16, 357-366. doi:10.1177/1362361311434545

Bowler, D.M., 1992. "Theory of Mind" in Asperger's Syndrome. J. Child Psychol. Psychiatry 33, 877893. doi:0021-9630/92

Callenmark, B., Kjellin, L., Rönnqvist, L., Bölte, S., 2013. Explicit versus implicit social cognition testing in autism spectrum disorder. Autism. doi:10.1177/1362361313492393

Carruthers, P., 2015. Mindreading in adults: evaluating two-systems views. Synthese. doi:10.1007/s11229-015-0792-3

Ciesielski, K.T., Courchesne, E., Elmasian, R., 1990. Effects of focused selective attention tasks on event-related potentials in autistic and normal individuals. Electroencephalogr. Clin. Neurophysiol. 75, 207-220. doi:10.1016/0013-4694(90)90174-I

Cohen, J., 1988. Statistical Power Analysis for the Behavioral Sciences. Routledge Academic, New York, NY.

Constantino, J.N., 2011. Autism as a quantitative trait, in: Amaral, D., Geschwind, D., Dawson, G. (Eds.), Autism Spectrum Disorders. Oxford University Press, New York, pp. 510-520. 
Constantino, J.N., Gruber, C.P., 2002. The social responsiveness scale. Western Psychological Services, Los Angeles, CA.

Constantino, J.N., Todd, R.D., 2003. Autistic Traits in the General Population: A Twin Study. Arch. Gen. Psychiatry 60, 524-530. doi:doi:10.1001/archpsyc.60.5.524

Decety, J., Lamm, C., 2007. The Role of the Right Temporoparietal Junction in Social Interaction: How Low-Level Computational Processes Contribute to Meta-Cognition. Neurosci. 13, 580-593. doi:10.1177/1073858407304654

Deschrijver, E., Bardi, L., Wiersema, J.R., Brass, M., 2015. Behavioral measures of implicit theory of mind in adults with high functioning autism. Cogn. Neurosci. 1-11. doi:10.1080/17588928.2015.1085375

Fisher, N., Happé, F.G.E., Dunn, J., 2005. The relationship between vocabulary, grammar, and false belief task performance in children with autistic spectrum disorders and children with moderate learning difficulties. J. Child Psychol. Psychiatry Allied Discip. 46, 409-419. doi:10.1111/j.14697610.2004.00371.x

Frith, U., 2004. Emanuel Miller lecture: Confusions and controversies about Asperger syndrome. J. Child Psychol. Psychiatry Allied Discip. 45, 672-686. doi:10.1111/j.1469-7610.2004.00262.x Frith, U., Happé, F., 1994. Autism: beyond "theory of mind." Cognition 50, 115-132.

Hudziak, J., Achenbach, T., Althoff, R., Pine, D., 2007. A dimensional approach to developmental psychopathology. Int. J. Methods Psychiatr. Res. 16, S16-S23. doi:10.1002/mpr

Ingersoll, B., Hopwood, C.J., Wainer, A., Donnellan, M.B., 2011. A comparison of three self-report measures of the broader autism phenotype in a non-clinical sample. J. Autism Dev. Disord. 41, 1646-1657. doi:10.1007/s10803-011-1192-2

Joshi, G., Wozniak, J., Petty, C., Martelon, M.K., Fried, R., Bolfek, A., Kotte, A., Stevens, J., Furtak, S.L., Bourgeois, M., Caruso, J., Caron, A., Biederman, J., 2013. Psychiatric comorbidity and functioning in a clinically referred population of adults with autism spectrum disorders: A comparative study. J. Autism Dev. Disord. 43, 1314-1325. doi:10.1007/s10803-012-1679-5

Kovács, Á.M., Kühn, S., Gergely, G., Csibra, G., Brass, M., 2014. Are All Beliefs Equal? Implicit Belief Attributions Recruiting Core Brain Regions of Theory of Mind. PLoS One 9, e106558. doi:10.1371/journal.pone.0106558

Kovács, Á.M., Téglás, E., Endress, A.D., 2010. The social sense: susceptibility to others' beliefs in human infants and adults. Science 330, 1830-4. doi:10.1126/science.1190792

Losh, M., Adolphs, R., Piven, J., 2011. The broad autism phenotype, in: Amaral, D., Geschwind, D., Dawson, G. (Eds.), Autism Spectrum Disorders. Oxford University Press, New York, pp. 457-476. Mannion, A., Leader, G., 2013. Comorbidity in autism spectrum disorder: A literature review. Res. Autism Spectr. Disord. 7, 1595-1616. doi:10.1016/j.rasd.2013.09.006 
Nijhof, A.D., Brass, M., Bardi, L., Wiersema, J.R., 2016. Measuring Mentalizing Ability: A WithinSubject Comparison between an Explicit and Implicit Version of a Ball Detection Task. PLoS One 11, e0164373. doi:10.1371/journal.pone.0164373

Onishi, K.H., Baillargeon, R., 2005. Do 15-month-old infants understand false beliefs? Science 308, 255-8. doi:10.1126/science.1107621

Ozonoff, S., Pennington, B.F., Rogers, S.J., 1991. Executive Function Deficits in High-Functioning Autistic Individuals: Relationship to Theory of Mind. J. Child Psychol. Psychiatry 32, 1081-1105. Parr, J., Le Couteur, A.S., 2013. “Broader Autism Phenotype," in: Volkmar, F.R. (Ed.), Encyclopedia of Autism Spectrum Disorders. Springer New York, p. 478. doi:10.1007/978-1-4614-6435-8

Pellicano, E., 2010. Individual differences in executive function and central coherence predict developmental changes in theory of mind in autism. Dev. Psychol. 46, 530-544. doi:10.1037/a0018287

Premack, D., Woodruff, G., 1978. Does the chimpanzee have a theory of mind? Behav. Brain Sci. 1, 515-526. doi:10.1017/S0140525X00076512

Rajendran, G., Mitchell, P., 2007. Cognitive theories of autism. Dev. Rev. 27, 224-260.

Robertson, A.E., Simmons, D.R., 2013. The relationship between sensory sensitivity and autistic traits in the general population. J. Autism Dev. Disord. 43, 775-784. doi:10.1007/s10803-012-1608-7

Robinson, E.B., Munir, K., Munaf, M.R., Hughes, M., McCormick, M.C., Koenen, K.C., 2011. Stability of autistic traits in the general population: Further evidence for a continuum of impairment. J. Am. Acad. Child Adolesc. Psychiatry 50, 376-384. doi:10.1016/j.jaac.2011.01.005

Ruzich, E., Allison, C., Smith, P., Watson, P., Auyeung, B., Ring, H., Baron-Cohen, S., 2015. Measuring autistic traits in the general population: a systematic review of the Autism-Spectrum Quotient (AQ) in a nonclinical population sample of 6,900 typical adult males and females. Mol. Autism 6, 2. doi:10.1186/2040-2392-6-2

Scheeren, A.M., de Rosnay, M., Koot, H.M., Begeer, S., 2013. Rethinking theory of mind in highfunctioning autism spectrum disorder. J. Child Psychol. Psychiatry 54, 628-35. doi:10.1111/jcpp.12007

Schmitz, N., Daly, E., Murphy, D., 2007. Frontal anatomy and reaction time in Autism. Neurosci. Lett. 412, 12-17. doi:10.1016/j.neulet.2006.07.077

Schneider, D., Slaughter, V.P., Bayliss, A.P., Dux, P.E., 2013. A temporally sustained implicit theory of mind deficit in autism spectrum disorders. Cognition 129, 410-7. doi:10.1016/j.cognition.2013.08.004

Schurz, M., Radua, J., Aichhorn, M., Richlan, F., Perner, J., 2014. Fractionating theory of mind: A meta-analysis of functional brain imaging studies. Neurosci. Biobehav. Rev. 42, 9-34. doi:10.1016/j.neubiorev.2014.01.009 
Schuwerk, T., Vuori, M., Sodian, B., 2015. Implicit and explicit Theory of Mind reasoning in autism spectrum disorders: The impact of experience. Autism 19, 459-468. doi:10.1177/1362361314526004

Senju, A., 2013. Atypical development of spontaneous social cognition in autism spectrum disorders. Brain Dev. 35, 96-101. doi:10.1016/j.braindev.2012.08.002

Senju, A., Southgate, V., Snape, C., Leonard, M., Csibra, G., 2011. Do 18-month-olds really attribute mental states to others? A critical test. Psychol. Sci. 22, 878-880. doi:10.1177/0956797611411584

Senju, A., Southgate, V., White, S., Frith, U., 2009. Mindblind eyes: an absence of spontaneous theory of mind in Asperger syndrome. Science (80-. ). 325, 883-5. doi:10.1126/science.1176170

Southgate, V., Senju, A., Csibra, G., 2007. Action anticipation through attribution of false belief by 2year-olds. Psychol. Sci. 18, 587-592. doi:10.1111/j.1467-9280.2007.01944.x

Spek, A.A., Scholte, E.M., Van Berckelaer-Onnes, I.A., 2010. Theory of mind in adults with HFA and asperger syndrome. J. Autism Dev. Disord. 40, 280-289. doi:10.1007/s10803-009-0860-y

Surian, L., Caldi, S., Sperber, D., 2007. Attribution of beliefs by 13-month-old infants. Psychol. Sci. 18, 580-6. doi:10.1111/j.1467-9280.2007.01943.x

Van Overwalle, F., 2009. Social cognition and the brain: a meta-analysis. Hum. Brain Mapp. 30, 82958. doi:10.1002/hbm.20547

Van Overwalle, F., Vandekerckhove, M., 2013. Implicit and explicit social mentalizing: dual processes driven by a shared neural network. Front. Hum. Neurosci. 7, 560.

doi:10.3389/fnhum.2013.00560

Wellman, H.M., Cross, D., Watson, J., 2001. Meta-analysis of theory-of-mind development: the truth about false belief. Child Dev. 72, 655-684. doi:10.1111/1467-8624.00304

Williams, D.M., Happé, F.G.E., 2009. What did I say? Versus what did I think? Attributing false beliefs to self amongst children with and without autism. J. Autism Dev. Disord. 39, 865-873. doi:10.1007/s10803-009-0695-6

Wimmer, H., Perner, J., 1983. Beliefs about beliefs: Representation and constraining function of wrong beliefs in young children's understanding of deception. Cognition 13, 103-128. 